\title{
BFKL pomeron in the external field of the nucleus in $(2+1)$-dimensional QCD
}

\author{
M.A.Braun, A.N.Tarasov \\ St.Petersburg State University, Russia
}

June 15, 2018

\begin{abstract}
The behaviour of the pomeron propagator in the external nuclear field is studied in the (2+1)-dimensional QCD. It is shown that in the physically interesting case when the field does not vanish at large rapidities the propagator in the field vanishes much faster than in the vacuum, in agreement with the results found in the local Regge-Gribov model. However if the nuclear field vanishes at high rapidities the field does not change the behaviour of the pomeron propagator.
\end{abstract}

\section{Introduction}

In the framework of the perturbative QCD at small $x$ and large $N_{c}$ the strong interaction is realized by the exchange of hard BFKL pomerons which interact via the 3-pomeron vertex governing their splitting and fusion. One can sum all the corresponding fan diagrams in the quasi-classical approximation by means of the Balitski-Kovchegov (BK) equation for $\gamma(\mathrm{h}) \mathrm{A}$ scattering [1,2] or by means of a pair of equations for AB scattering introduced in [3]. With the growth of energy the role of pomeron loops becomes important and one has to search for methods to take them into account. In our previous publications we noted that calculations of loops may become easier if one starts with the perturbative approach inside the nucleus from the start. At least in the local Regge-Gribov model the nuclear field transforms the supercritical pomeron with the intercept greater than unity into a subcritical one with the intercept smaller than unity [4]. As a result with the growth of energy the loop becomes relatively smaller, so that it can be calculated perturbatively.

This result can be easily understood from the structure of the model in the nuclear surrounding. The Lagrangian of the Regge-Gribov model has a form

$$
\mathcal{L}=\Phi^{\dagger}\left(\frac{\partial}{\partial y}+H\right) \Phi+V \Phi^{\dagger} \Phi\left(\Phi^{\dagger}+\Phi\right)
$$

where $\Phi$ and $\Phi^{\dagger}$ are quantum fields which describe the pomeron, $H$ is the free "Hamiltonian" and $V$ - the 3-pomeron vertex. In the quasi-classical approximation one has $\Phi^{\dagger}=0$ and $\Phi=\Phi_{0}$, the latter given by the sum of all fan diagrams corresponding to the interaction with the nucleus. To calculate quantum correction one can shift the field $\Phi \rightarrow \Phi+\Phi_{0}$ and thus study the model in the nuclear background field $\Phi_{0}$. This introduces a new interaction $V \Phi_{0} \Phi^{\dagger^{2}}$ corresponding to the annihilation of a pair of pomerons and, most important, the pomeron propagator must now be calculated in the background field $\Phi_{0}$. It turns out that this propagator, which in the vacuum exponentially grows with rapidity, in the background nuclear field exponentially vanishes. As a result in the background field pomeron loops do not grow with rapidity and can be easily controlled. 
Of course the problem is to see if this result can be generalized to the perturbative QCD pomeron. Our numerical calculations have shown that the QCD pomeron propagator in the external field of the nucleus also vanishes at high rapidities in contrast to its behaviour in the vacuum [5]. Unfortunately the realistic QCD in 3+1 dimensions does not allow to confirm this result analytically, since both the relevant BK equation and the BFKL equation in the external field do not allow for analytic solutions. As was shown a some time ago the situation is improved in the (2+1)-dimensional QCD, where a solution to the BK equation can be obtained in a form which analytically shows its rapidity dependence [6].

In this note we use this known solution to study the behaviour of the BFKL propagator in the external field given by this solution and corresponding to the field inside a big nucleus. Our results show that this behaviour, as well as the behaviour of the nuclear field itself, very much depend on the initial conditions for evolution. If they are chosen in such a way that at large rapidities the fan-diagram-field tends to unity (the dipole $S$-matrix vanishes) then solutions of the BFKL equation in this field also vanish in this limit. However for different initial conditions, for which the fan diagram-field vanishes at large rapidities (the dipole $S$-matrix tends to unity), this field does not change the behaviour of the solutions of the BFKL equation. A striking result of our study is that the interval of the coordinates in which the BFKL propagator in the external field is different from zero diminishes with the fall of energy. As a consequence pomerons cannot form loops in the nuclear field. This implies that the quasi-classical approach gives the complete solution to the quantum field theory of interacting pomerons in the nuclear field in $2+1$ dimensions.

\section{Main equations}

As found in [6] the kernel of the BK equation (the rescaled triple pomeron vertex) is changed for the $(2+1)$-dimensional space according to

$$
\frac{\alpha_{s} N_{c}}{2 \pi^{2}} \frac{r_{21}^{2}}{r_{23}^{2} r_{31}^{2}} \rightarrow 2 \alpha_{s} N_{c} \theta\left(r_{2,1}^{\max }-r_{3}\right) \theta\left(r_{3}-r_{2,1}^{\min }\right)
$$

where $r_{2,1}^{\max (\min )}$ is the maximal (minimal) of the coordinates $r_{2}$ and $r_{1} ; r_{21}=r_{2}-r_{1}$ etc. This vertex is a constant different from zero only when $r_{3}$ lies between $r_{2}$ and $r_{1}$. This allows to write the BK equation in a simple form. Let $S_{r_{2} r_{1}}(y)$ be the $S$-matrix for the interaction of a dipole stretched between spatial (1-dimensional) points $r_{2}>r_{1}$ at rapidity $Y$. The BK equation for $S$, which sums all the fan diagrams, is

$$
\frac{\partial S_{r_{2} r_{1}}}{\partial y}=\int_{r_{1}}^{r_{2}} d r_{0}\left(S_{r_{2} r_{0}} S_{r_{0} r_{1}}-S_{r_{2} r_{1}}\right), \quad y=2 \pi \bar{\alpha} Y,
$$

where $Y$ is the rapidity. This equation can be further simplified if we introduce a function

$$
\Psi_{r_{2} r_{1}}(y)=e^{r_{21} y} S_{r_{2} r_{1}}, \quad r_{21}=r_{2}-r_{1} \geq 0 .
$$

The equation for $\Psi$ is

$$
\frac{\partial \Psi_{r_{2} r_{1}}}{\partial y}=\int_{r_{1}}^{r_{2}} d r_{0} \Psi_{r_{2} r_{0}} \Psi_{r_{0} r_{1}}
$$

Considering $\Psi_{r_{2} r_{1}}(y)$ as a triangular matrix

$$
\Psi_{r_{2} r_{1}}(y)=<r_{2}|\Psi(y)| r_{1}>, \quad r_{2} \geq r_{1},
$$

we can rewrite Eq. (5) as

$$
\frac{\partial \Psi(y)}{\partial y}=\Psi^{2}(y)
$$


with a solution satisfying the initial condition

$$
\left.\Psi_{r_{2} r_{1}}(y)\right|_{y=0}=\left.S_{r_{2} r_{1}}(y)\right|_{y=0}=\Psi_{r_{2} r_{1}}(0)
$$

and given by the matrix formula

$$
\Psi(y)=\Psi(0)[1-y \Psi(0)]^{-1} .
$$

To obtain the equation for the BFKL pomeron inside the nucleus we first rewrite Eq. (3) in terms of the scattering amplitude $\Phi_{r_{2} r_{1}}(y)$ defined by

$$
S_{r_{2} r_{1}}(y)=1-\Phi_{r_{2} r_{1}}(y) .
$$

We get

$$
\frac{\partial \Phi_{r_{2} r_{1}}}{\partial y}=\int_{r_{1}}^{r_{2}} d r_{0}\left(\Phi_{r_{2} r_{0}}+\Phi_{r_{0} r_{1}}-\Phi_{r_{2} r_{1}}-\Phi_{r_{2} r_{0}} \Phi_{r_{0} r_{1}}\right)
$$

This scattering amplitude represents the sum of fan diagrams connecting the projectile with the nucleus target. This is precisely the field created by the nuclear background.

Dropping the non-linear term one obtains the BFKL equation in $2+1$ dimensions in the vacuum

$$
\frac{\partial P_{r_{2} r_{1}}}{\partial y}=\int_{r_{1}}^{r_{2}} d r_{0}\left(P_{r_{2} r_{0}}+P_{r_{0} r_{1}}-P_{r_{2} r_{1}}\right) .
$$

The BFKL equation in the nuclear field is obtained when one adds to the right-hand side terms which correspond to the interaction of the pomeron with the nuclear field via the triple pomeron vertex

$$
\frac{\partial P_{r_{2} r_{1}}}{\partial y}=\int_{r_{1}}^{r_{2}} d r_{0}\left(P_{r_{2} r_{0}}+P_{r_{0} r_{1}}-P_{r_{2} r_{1}}-\Phi_{r_{2} r_{0}} P_{r_{0} r_{1}}-P_{r_{2} r_{0}} \Phi_{r_{0} r_{1}}\right) .
$$

Expressing $\Phi$ via $S$ we rewrite this equation as

$$
\frac{\partial P_{r_{2} r_{1}}}{\partial y}=\int_{r_{1}}^{r_{2}} d r_{0}\left(S_{r_{2} r_{0}} P_{r_{0} r_{1}}+P_{r_{2} r_{0}} S_{r_{0} r_{1}}-P_{r_{2} r_{1}}\right)
$$

Finally we introduce

$$
Q_{r_{2} r_{1}}(y)=e^{r_{21} y} P_{r_{2} r_{1}}(y),
$$

for which the equation simplifies to

$$
\frac{\partial Q_{r_{2} r_{1}}}{\partial y}=\int_{r_{1}}^{r_{2}} d r_{0}\left(\Psi_{r_{2} r_{0}} Q_{r_{0} r_{1}}+Q_{r_{2} r_{0}} \Psi_{r_{0} r_{1}}\right)
$$

or in the matrix notation

$$
\frac{\partial Q(y)}{\partial y}=\{Q(y), \Psi(y)\}
$$

Solution to this equation with the initial condition

$$
\left.Q\left(y, y^{\prime}\right)\right|_{y=y^{\prime}}=Q\left(y^{\prime}, y^{\prime}\right)
$$

is found to be

$$
\begin{aligned}
Q\left(y, y^{\prime}\right) & =\Psi^{-1}\left(y^{\prime}\right) \Psi(y) Q\left(y^{\prime}, y^{\prime}\right) \Psi(y) \Psi^{-1}\left(y^{\prime}\right) \\
& \equiv T\left(y, y^{\prime}\right) Q\left(y^{\prime} y^{\prime}\right) T\left(y, y^{\prime}\right),
\end{aligned}
$$

where we define triangular matrix

$$
T\left(y, y^{\prime}\right)=\Psi^{-1}\left(y^{\prime}\right) \Psi(y)=\Psi(y) \Psi^{-1}\left(y^{\prime}\right) .
$$


Note that $\Psi(y)$ and $\Psi^{-1}\left(y^{\prime}\right)$ commute at any $y$ and $y^{\prime}$, since they both are functions of the same matrix $\Psi(0)$.

In the following we shall consider a simple case when the nuclear 1-dimensional density is constant ("nuclear matter"). Then the initial BK function $\Psi_{r_{2} r_{1}}(0)$ will depend only on the distance between the gluons

$$
\Psi_{r_{2} r_{1}}(0)=\Psi_{r_{21}}(0) .
$$

As a result $\Psi_{r_{2} r_{1}}(y)$ will depend only on the distance $\rho_{21}$ at all values of $y$ and consequently $T_{r_{2} r_{1}}\left(y, y^{\prime}\right)$ will also depend only on the distance $r_{21}$. As a matrix

$$
T_{r_{2} r_{1}}\left(y, y^{\prime}\right)=\theta\left(r_{21}\right) T_{r_{21}}\left(y, y^{\prime}\right) .
$$

\section{The Green function of the BFKL equation (14)}

We shall first study the Green function $G_{r_{2} r_{1} \mid r_{2}^{\prime} r_{1}^{\prime}}\left(y, y^{\prime}\right)$ which satisfies

$$
G_{r_{2} r_{1} \mid r_{2}^{\prime} r_{1}^{\prime}}\left(y^{\prime}, y^{\prime}\right)=\delta\left(r_{2}-r_{2}^{\prime}\right) \delta\left(r_{1}-r_{1}^{\prime}\right)
$$

and we assume that $r_{2}=\max \left\{r_{2}, r_{1}\right\}$ and $r_{2}^{\prime}=\max \left\{r_{2}^{\prime}, r_{1}^{\prime}\right\}$. Note that this is not the pomeron propagator, which will be studied later. To find $G$ we have to search for a solution of the equation in variables $r_{2}$ and $r_{1}$ for $P$ which satisfies (22). In terms of

$$
F_{r_{2} r_{1}}\left(y, y^{\prime}\right)=e^{y r_{21}} G_{r_{2} r_{1} \mid r_{2}^{\prime} r_{1}^{\prime}}\left(y, y^{\prime}\right)
$$

we have to find a solution of Eq. (14) which satisfies

$$
F_{r_{2} r_{1}}\left(y^{\prime}, y^{\prime}\right)=e^{y^{\prime} r_{21}^{\prime}} \theta\left(r_{21}\right) \delta\left(r_{2}-r_{2}^{\prime}\right) \delta\left(r_{1}-r_{1}^{\prime}\right) \text {. }
$$

Obviously we should also have $r_{2}^{\prime} \geq r_{1}^{\prime}$.

According to (18) it is given by

$$
\begin{gathered}
F_{r_{2} r_{1}}\left(y, y^{\prime}\right)=e^{y^{\prime} r_{21}^{\prime}} \theta\left(r_{21}\right) \theta\left(r_{21}^{\prime}\right) \int d r_{0} d r_{0}^{\prime} T_{r_{2} r_{0}}\left(y, y^{\prime}\right) \delta\left(r_{0}-r_{2}^{\prime}\right) \delta\left(r_{0}^{\prime}-r_{1}^{\prime}\right) T_{r_{0}^{\prime} r_{1}}\left(y, y^{\prime}\right) \\
=e^{y^{\prime} r_{21}^{\prime}} \theta\left(r_{21}\right) \theta\left(r_{21}^{\prime}\right) T_{r_{2} r_{2}^{\prime}}\left(y, y^{\prime}\right) T_{r_{1}^{\prime} r_{1}}\left(y, y^{\prime}\right) .
\end{gathered}
$$

The Green function itself will be given by

$$
G_{r_{2} r_{1} \mid r_{2}^{\prime} r_{1}^{\prime}}\left(y, y^{\prime}\right)=e^{y^{\prime} r_{21}^{\prime}-y r_{21}} \theta\left(r_{21}\right) \theta\left(r_{21}^{\prime}\right) T_{r_{2} r_{2}^{\prime}}\left(y, y^{\prime}\right) T_{r_{1}^{\prime} r_{1}}\left(y, y^{\prime}\right) \text {. }
$$

This expression is different from zero only in the interval

$$
G_{r_{2} r_{1} \mid r_{2}^{\prime} r_{1}^{\prime}}\left(y, y^{\prime}\right) \neq 0 \text { only if } r_{1}<r_{1}^{\prime}<r_{2}^{\prime}<r_{2} .
$$

Here $r_{2}$ and $r_{2}^{\prime}$ are the maximal coordinates of the initial and final gluons and $r_{1}^{\prime}$ and $r^{\prime}$ are their minimal coordinates. From (25) one concludes that the interval of coordinates where $G$ is different from zero is restricted on both sides. This interval at $y$ should be wholly inside the one at the initial rapidity $y^{\prime}$. This remarkable property will have far-reaching consequences for the whole model, as will be discussed in the following.

For the considered case of nuclear matter when $T_{r_{2} r_{1}}=T_{r_{21}}$ the Green function $G$ can be presented in the form which explicitly shows its symmetry in the initial and final gluons

$$
G_{r_{2} r_{1} \mid r_{2}^{\prime} r_{1}^{\prime}}\left(y, y^{\prime}\right)=e^{y^{\prime} s^{\prime}-y s} T_{s_{2}}\left(y, y^{\prime}\right) T_{s_{1}}\left(y, y^{\prime}\right) \text {. }
$$


where

$$
\begin{aligned}
s & =\max \left\{r_{2}, r_{1}\right\}-\min \left\{r_{2}, r_{1}\right\} \quad s^{\prime}=\max \left\{r_{2}^{\prime}, r_{1}^{\prime}\right\}-\min \left\{r_{2}^{\prime}, r_{1}^{\prime}\right\}, \\
s_{2} & =\max \left\{r_{2}, r_{1}\right\}-\max \left\{r_{2}^{\prime}, r_{1}^{\prime}\right\}, \quad s_{1}=\min \left\{r_{2}^{\prime}, r_{1}^{\prime}\right\}-\min \left\{r_{2}, r_{1}\right\}
\end{aligned}
$$

and $s, s^{\prime}, s_{1}$ and $s_{2}$ are all to be non-negative so that $s_{2}+s_{1}=s-s^{\prime} \geq 0$.

At $y^{\prime}=0$ we have

$$
T_{r_{2} r_{1}}(y, 0)=\left([1-y \Psi(0)]^{-1}\right)_{r_{2} r_{1}} .
$$

From the equation

$$
\int_{r_{1}}^{r_{2}} d r_{0} T_{r_{2} r_{0}}(y, 0)\left(\delta\left(r_{0}-r_{1}\right)-y \Psi_{r_{0} r_{1}}(0)\right)=\delta\left(r_{2}-r_{1}\right)
$$

we conclude that

$$
T_{r_{2} r_{1}}(y, 0)=\delta\left(r_{2}-r_{1}\right)+Y_{r_{2} r_{1}}(y)
$$

where $Y(y)$ is a smooth function of $r_{2}$ and $r_{1}$. Presence of the $\delta$-function allows to get the correct right-hand side, which otherwise would be zero at $r_{2}=r_{1}$. Using the fact that the BK solution depends only on the difference $r_{21}$ we find

$$
T_{r_{21}}(y)=\delta\left(r_{21}\right)+Y_{r_{21}}(y)
$$

To find $Y_{r}(y)$ we set up an evolution equation in $y$. Obviously

$$
\frac{\partial T_{r}(y, 0)}{\partial y}=\left(\Psi(0) T^{2}(y, 0)\right)_{r}=\int_{0}^{r} d r_{1} \Psi_{r-r_{1}}(0) \int_{0}^{r_{1}} d r_{2} T_{r_{1}-r_{2}}(y, 0) T_{r_{2}}(y, 0),
$$

or in terms of $Y$

$$
\frac{\partial Y_{r}(y)}{\partial y}=\Psi_{r}(0)+2 \int_{0}^{r} d r_{1} \Psi_{r-r_{1}}(0) Y_{r_{1}}(y)+\int_{0}^{r} d r_{1} \Psi_{r-r_{1}}(0) \int_{0}^{r_{1}} d r_{2} Y_{r_{1}-r_{2}}(y) Y_{r_{2}}(y)
$$

with the initial condition

$$
Y_{r}(0)=0
$$

From this evolution equation one can numerically find $Y_{r}(y)$ at any $y$ by the standard RungeKutta procedure. At arbitrary $y$ and $y^{\prime}$

$$
T\left(y, y^{\prime}\right)=\left[1-y^{\prime} \Psi(0)\right][1-y \Psi(0)]^{-1} .
$$

From this we find

$$
T_{r}\left(y, y^{\prime}\right)=\delta(r)+X_{r}\left(y, y^{\prime}\right)
$$

where $X\left(y, y^{\prime}\right)$ is a smooth function linear in $y^{\prime}$ :

$$
X_{r}\left(y, y^{\prime}\right)=Y_{r}(y)-y^{\prime}\left[\Psi_{r}(0)+\int_{0}^{r} d r^{\prime} \Psi_{r-r^{\prime}} Y_{r^{\prime}}(y)\right] .
$$

In absence of the interaction with the nucleus $S$-matrix corresponding to the BK equation turns into unity. In terms of the triangular matrices it implies

$$
S_{r_{2} r_{1}}^{(0)}(y)=\theta\left(r_{2}-r_{1}\right)
$$

and does not depend on $y$. From this we find in the vacuum

$$
T_{r}^{(0)}\left(y, y^{\prime}\right)=\delta(r)+\left(y-y^{\prime}\right) e^{y r} \theta(r) .
$$

Using this and (24) we obtain the Green function $G^{(0)}$ in the vacuum:

$$
\begin{gathered}
G_{r_{2} r_{1} \mid r_{2}^{\prime} r_{1}^{\prime}}^{(0)}\left(y, y^{\prime}\right)=e^{-\left(y-y^{\prime}\right) r_{21}^{\prime}}\left\{\delta\left(r_{22^{\prime}}\right) \delta\left(r_{1^{\prime} 1}\right)\right. \\
\left.+\left(y-y^{\prime}\right) \delta\left(r_{1^{\prime} 1}\right) \theta\left(r_{22^{\prime}}\right)+\left(y-y^{\prime}\right) \delta\left(r_{22^{\prime}}\right) \theta\left(r_{1^{\prime} 1}\right)+\left(y-y^{\prime}\right)^{2} \theta\left(r_{22^{\prime}}\right) \theta\left(r_{1^{\prime} 1}\right)\right\}
\end{gathered}
$$

where it is assumed that $r_{22^{\prime}}, r_{1^{\prime} 1} \geq 0$. This expression coincides with the one previously obtained in [6]. As expected in absence of the external field the Green function depends only on the difference $y-y^{\prime}$. 


\section{Pomeron propagator in the nuclear field}

In contrast with the Green function $G$ defined by the equation

$$
\left(\frac{\partial}{\partial y}+H\right) G=1
$$

where $H$ is the BFKL Hamiltonian in the external field, the pomeron propagator $g$ is defined by

$$
\left(\frac{\partial}{\partial y}+H\right) g=p_{1}^{-2} p_{2}^{-2}
$$

so that it is related to the Green function considered previously as

$$
g\left(r_{2}, r_{1} \mid r_{2}^{\prime}, r_{1}^{\prime}\right)=\nabla_{1}^{-2} \nabla_{2}^{-2} G\left(r_{2}, r_{1} \mid r_{2}^{\prime}, r_{1}^{\prime}\right)=G\left(r_{2}, r_{1} \mid r_{2}^{\prime}, r_{1}^{\prime}\right) \nabla_{1^{\prime}}^{-2} \nabla_{2^{\prime}}^{-2},
$$

or equivalently

$$
\nabla_{1}^{2} \nabla_{2}^{2} g\left(r_{2}, r_{1} \mid r_{2}^{\prime}, r_{1}^{\prime}\right)=g\left(r_{2}, r_{1} \mid r_{2}^{\prime}, r_{1}^{\prime}\right) \nabla_{1}^{\prime 2} \nabla_{2}^{\prime 2}=G\left(r_{2}, r_{1} \mid r_{2}^{\prime}, r_{1}^{\prime}\right)
$$

where in the last equality the derivatives act on the left. Obviously relations (44) determine $g$ non-uniquely. One can add to it any function linear in $r_{2}-r_{2}^{\prime}$ or in $r_{1}-r_{1}^{\prime}$. Our choice will consist in selecting $g$ to be different from zero in the same intervals of initial and final gluon coordinates as $G$, namely $s, s^{\prime}, s_{1}, s_{2} \geq 0$ where $s$ are defined by (27). As we shall find the propagator $g$ defined in this way at $y>y^{\prime}$ vanishes at large separation of points. Linear terms that can be added to it and different from zero outside the interval (25) violate this condition and seem to us unacceptable on formal grounds, since correspond to strong infrared singularities at all values of $y$.

To make our choice we notice that in the one-dimensional transverse coordinate space function $h(r)=\nabla^{-2} \delta(r)$ can be presented as

$$
h(r)=r \theta(r) .
$$

Indeed we have

$$
\nabla^{2} h(r)=h^{\prime \prime}(r)=(r \theta(r))^{\prime \prime}=r \delta^{\prime}(r)+2 \delta(r)=\delta(r) .
$$

This implies that the Green function $g\left(y, r_{2}, r_{1} \mid y^{\prime}, r_{2}^{\prime}, r_{1}^{\prime}\right)$ may be chosen to satisfy the BFKL equation

$$
\left(\frac{\partial}{\partial y}+H\right) g\left(y, r_{2}, r_{1} \mid y^{\prime}, r_{2}^{\prime}, r_{1}^{\prime}\right)=\left(r_{2}-r_{2}^{\prime}\right) \theta\left(r_{2}-r_{2}^{\prime}\right)\left(r_{1}^{\prime}-r_{1}\right) \theta\left(r_{1}^{\prime}-r_{1}\right)
$$

where it is assumed $r_{21}, r_{21}^{\prime} \geq 0$. Note the inverse order of the initial and final coordinates in the second factor on the right. It is this order that guarantees the desired property of $g$ to be different from zero only in the interval (25).

We can solve this equation in the same manner as when searching for the Green function $G$. We introduce matrix

$$
f_{r_{2} r_{1}}\left(y, y^{\prime}\right)=\theta\left(r_{21}\right) e^{y r_{21}} g_{r_{2} r_{1} \mid r_{2}^{\prime} r_{1}^{\prime}}\left(y, y^{\prime}\right)
$$

with $r_{21}, r_{21}^{\prime} \geq 0$. It will satisfy Eq. (46) with the initial condition

$$
f_{r_{2} r_{1}}\left(y^{\prime}, y^{\prime}\right)=\theta\left(r_{21}\right) e^{y^{\prime} r_{21}} \theta\left(r_{21}^{\prime}\right)\left(r_{2}-r_{2}^{\prime}\right) \theta\left(r_{2}-r_{2}^{\prime}\right)\left(r_{1}^{\prime}-r_{1}\right) \theta\left(r_{1}^{\prime}-r_{1}\right) .
$$

According to Eq. (18) the solution is given by

$$
f_{r_{2} r_{1}}\left(y, y^{\prime}\right)=\sum_{r_{0}, r_{0}^{\prime}} \theta\left(r_{00^{\prime}}\right) T_{r_{2} r_{0}}\left(y, y^{\prime}\right) r_{02^{\prime}} \theta\left(r_{02^{\prime}}\right) r_{10}^{\prime} \theta\left(r_{10}^{\prime}\right) e^{y^{\prime} r_{00^{\prime}}} T_{r_{0}^{\prime} r_{1}}\left(y, y^{\prime}\right),
$$


where we use the notation $r_{00^{\prime}}=r_{0}-r_{0}^{\prime}$ and $r_{10}^{\prime}=r_{1}^{\prime}-r_{0}^{\prime}$.

Taking into account that $T_{r_{2} r_{1}}$ depends only on the distance $r_{21}$ between the points we find

$$
\begin{gathered}
g_{r_{2} r_{1} \mid r_{2}^{\prime} r_{1}^{\prime}}\left(y, y^{\prime}\right)=\theta\left(r_{21}\right) \theta\left(r_{21}^{\prime}\right) e^{-r_{21}\left(y-y^{\prime}\right)} \int d r \int d r^{\prime} \theta\left(r_{21}-r-r^{\prime}\right) \theta\left(r_{22^{\prime}}-r\right) \theta\left(r_{1^{\prime} 1}-r^{\prime}\right) \\
e^{-y^{\prime}\left(r+r^{\prime}\right)}\left(r_{22^{\prime}}-r\right)\left(r_{1^{\prime} 1}-r^{\prime}\right) T_{r}\left(y, y^{\prime}\right) T_{r^{\prime}}\left(y, y^{\prime}\right) .
\end{gathered}
$$

Integration over $r$ and $r^{\prime}$ is restricted by the last two $\theta$-functions to the interval

$$
0<r<r_{22^{\prime}}, \quad O<r^{\prime}<r_{1^{\prime} 1} .
$$

From this we conclude

$$
r+r^{\prime}<r_{22^{\prime}}+r_{1^{\prime} 1}=r_{21}-r_{21}^{\prime}<r_{21}
$$

so that $\theta\left(r_{21}-r-r^{\prime}\right)=1$. As a result this expression factorizes

$$
g_{r_{2} r_{1} \mid r_{2}^{\prime} r_{1}^{\prime}}\left(y, y^{\prime}\right)=e^{-r_{21}\left(y-y^{\prime}\right)} U_{r_{22^{\prime}}}\left(y, y^{\prime}\right) U_{r_{1^{\prime} 1}}\left(y, y^{\prime}\right),
$$

where we have defined a matrix

$$
U_{r}\left(y, y^{\prime}\right)=\int_{0}^{r} d r^{\prime}\left(r-r^{\prime}\right) T_{r^{\prime}}\left(y, y^{\prime}\right) e^{-y^{\prime} r^{\prime}} .
$$

In the notation symmetric in the initial and final gluons this can be rewritten as

$$
g_{r_{2} r_{1} \mid r_{2}^{\prime} r_{1}^{\prime}}\left(y, y^{\prime}\right)=e^{-s\left(y-y^{\prime}\right)} U_{s_{1}}\left(y, y^{\prime}\right) U_{s_{2}}\left(y, y^{\prime}\right) .
$$

where $s, s^{\prime}, s_{1}$ and $s_{2}$ are defined by Eqs. (27).

We have seen that

$$
T\left(y, y^{\prime}\right)=\left[1-y^{\prime} \Psi(0)\right][1-y \Psi(0)]^{-1}
$$

and that the second factor contains a $\delta$-contribution

$$
[1-y \Psi(0)]^{-1}=1+Y(y) \text {. }
$$

From this we find

$$
T\left(y, y^{\prime}\right)=1+X\left(y, y^{\prime}\right), \quad X\left(y, y^{\prime}\right)=Y(y)-y^{\prime} \Psi(0)-y^{\prime} \Psi(0) Y(y),
$$

so that $T_{r_{2} r_{1}}$ contains a $\delta$ term. If $T_{r_{2} r_{1}}$ depends only on the distance $r_{21}$ we find

$$
T_{r}\left(y, y^{\prime}\right)=\delta(r)+X_{r}\left(y, y^{\prime}\right),
$$

where $X_{r}\left(y, y^{\prime}\right)$ is a smooth function of $r$ linear in $y^{\prime}$ :

$$
X_{r}\left(y, y^{\prime}\right)=Y_{r}(y)-y^{\prime}\left[\Psi_{r}(0)+\int_{0}^{r} d r^{\prime} \Psi_{r-r^{\prime}} Y_{r^{\prime}}(y)\right] .
$$

Separating the $\delta$ function from $T$ one gets

$$
U_{r}\left(y, y^{\prime}\right)=r+\int_{0}^{r} d r^{\prime} X_{r^{\prime}}\left(y, y^{\prime}\right)\left(r-r^{\prime}\right) e^{-y^{\prime} r^{\prime}} .
$$

In absence of the nuclear field, using (39) one obtains

$$
U_{r}^{(0)}\left(y-y^{\prime}\right)=\frac{e^{\left(y-y^{\prime}\right) r}-1}{y-y^{\prime}} .
$$

From this using (51) we find the vacuum propagator, which naturally depends only on the difference $y-y^{\prime}$, which we denote as simply $y$

$$
g_{r_{2} r_{1} \mid r_{2}^{\prime} r_{1}^{\prime}}(y)=\frac{1}{y}\left\{e^{-y r_{21}}+e^{-y r_{21}^{\prime}}-e^{-y r_{2^{\prime} 1}}-e^{-y r_{21^{\prime}}}\right\},
$$

where it is assumed that $r_{1} \leq r_{1}^{\prime} \leq r_{2}^{\prime} \leq r_{2}$. 


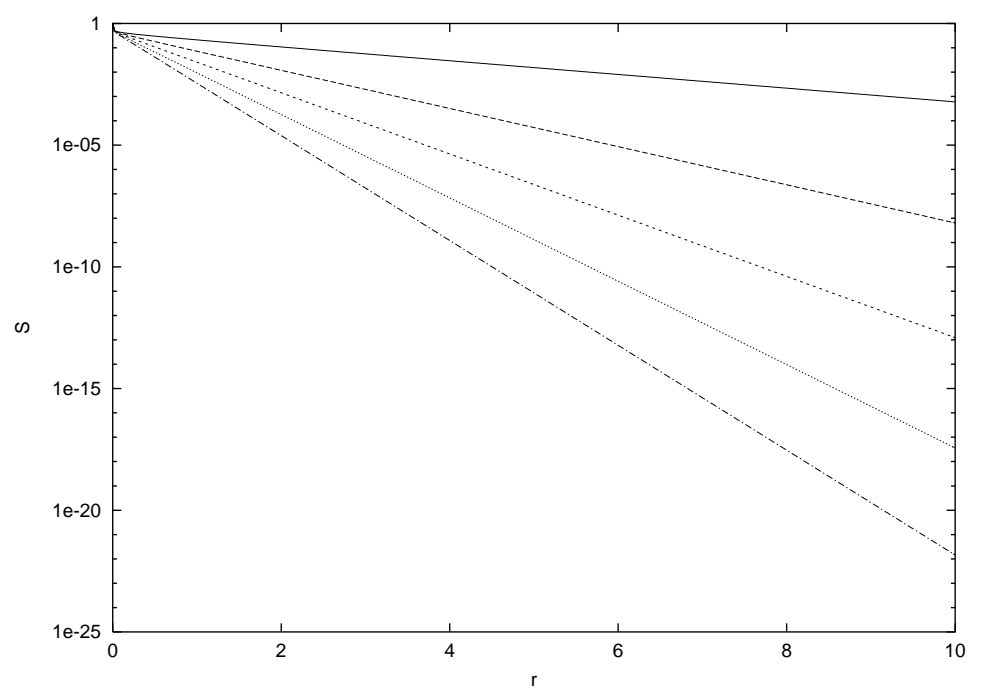

Figure 1: $S$-matrix $S_{r}(y)$ evolved by the BK equation from the initial function Eq. (64) with $\gamma=0.1$ as a function of $r$. Curves from top to bottom correspond to $y=1,3,5,7$ and 9

\subsection{Numerical results}

Both the nuclear field characterized by the BK $S$-matrix $S_{r_{2} r_{1}}(y)$ and the pomeron propagator are determined by the coupling to the nucleus contained in the BK $S$-matrix at $y=0$. In the nuclear matter on physical grounds at $r=0$ one requires $S_{r}(0)_{r=0}=1$ corresponding to vanishing of integration for zero-dimensional dipoles. As noted in [6] the behaviour of $S$ with the growth of energy crucially depends on the behaviour of $S_{r}(0) S_{r}(0)_{r \rightarrow 0} \sim 1-c r^{\gamma}$. In particular for $\gamma<1$ it was found that the asymptotical behavior at $y>>1$ was

$$
S_{r}(y) \sim e^{-c \Gamma(1+\gamma) y^{1-\gamma} r} .
$$

On the other hand for $\gamma>1$ the $S$-matrix was expected to tend to unity in the large region $r<<y^{\gamma-1}$.

In our calculations we have taken $S_{r}(0)$ in the form

$$
S_{r}(0)=e^{-r^{\gamma}}
$$

Numerical results show that for $\gamma<1$ the asymptotical behaviour (62) is well fulfilled. In Fig. 1 we show the behavour $S_{r}(y)$ with $\gamma=0.1$ for values of rescaled rapidity $y=1,3,5,7$ and 9 . In fact they are well described by (62) practically at all values of $r$ and $y$. However for $\gamma>1$ we find the asymptotical behaviour somewhat different from what was expected in [6]. In Fig. 2 we present $S_{r}(y)$ with $\gamma=2$ at the same rapidities $y=1,3,5,7$ and 9 . They all fall onto the exponential dependence on $r$

$$
S_{r}(r) \sim e^{-b(y) r} .
$$

In contrast to the case $\gamma<1$ the slope $b(y)$ falls with $y$ but not to zero but to the finite value $\sim 0.33$. The same picture is observed with still higher values of $\gamma$.

Passing to the pomeron propagator we note that it depends on three independent variables. Assuming that $r_{1} \leq r_{1}^{\prime} \leq r_{2}^{\prime} \leq r_{2}$, they can be taken as any three of the four differences $r_{21}, r_{21}^{\prime}$, $r_{22^{\prime}}$ and $r_{1^{\prime} 1}$, taking non-negative values and constrained by the relation

$$
r_{21}^{\prime}+r_{22^{\prime}}+r_{1^{\prime} 1}=r_{21}
$$




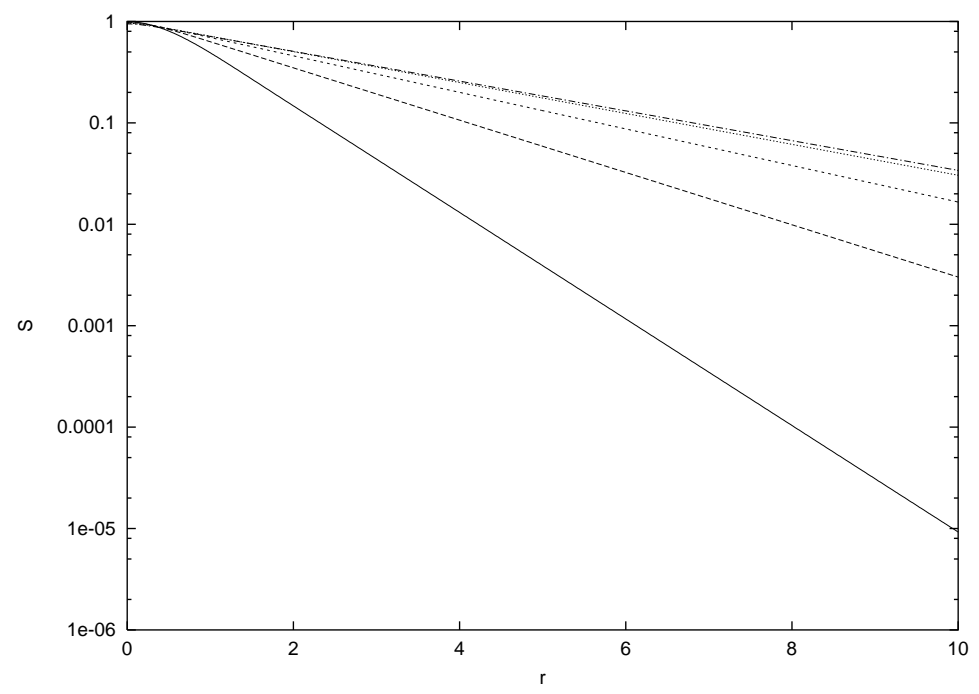

Figure 2: Same as Fig. 1 with $\gamma=2.0$. Curves from bottom to top correspond to $y=1,3,5,7$ and 9

To see both $r$ - and $y$ - dependence we shall first study integrals of $g$ at $y^{\prime}=0$ over $r_{22^{\prime}}$ and $r_{1^{\prime} 1}$ with fixed $r_{21}=r$

$$
g_{1}(y, r)=e^{-y r} \int_{0}^{r} d r_{1} U_{r_{1}}(y, 0) \int_{0}^{r-r_{1}} d r_{2} U_{r_{2}}(y, 0)
$$

and with fixed $r_{21}^{\prime}=r$

$$
\left.g_{2}(y, r)\right)=e^{-y r} \int_{0}^{r_{m}-r} d r_{1} U_{r_{1}}(y, 0) e^{-y r_{1}} \int_{0}^{r_{m}-r-r_{1}} d r_{2} U_{r_{2}}(y, 0) e^{-y r_{2}}
$$

where $r_{m}$ is the upper limit of the integration, (formally infinite, but finite in the course of calculations and taken $r_{m}=20$ ).

Our numerical results for $g_{r}^{(1)}$ and $g_{r}^{(2)}$ with $\gamma=0.1$ and $y=1,3,5,7$ and 9 are shown in Figs. 3 and 4 respectively. Similar results with $\gamma=2.0$ are illustrated in Figs. 5 and 6 . To compare in Fig. 7 and 8 we plot the same quantities for the vacuum case. In all cases the integrated propagator diminishes with energy and coordinate.

To clearly see the influence of the field and the role of value of $\gamma$ in Fig. 9 we show values of $g(y, 0)$ integrated over all three independent variables from (65). To facilitate the comparison results with $\gamma=0.1$ and $\gamma=2.0$ were rescaled to coincide with the vacuum result at $y=1$. We observe a clear distinction between the cases $\gamma<1$ and $\gamma>1$ previously stressed in [6]. With $\gamma<1$ we find that the propagator in the nuclear field indeed goes to zero much faster than in the vacuum as advocated in our previous studies in the $3+1$ dimensional case. In contrast with $\gamma>1$ we do not observe any influence of the field. This goes in line with the assertion in [6] that this case corresponds to asymptotic vanishing of the dipole interaction.

\section{Loops}

In $3+1$ dimensions in the theory of interacting BFKL pomerons the interaction is given by

$$
S_{I}=\frac{2 \alpha_{s}^{2} N_{c}}{\pi} \int d y \frac{d^{2} r_{1} d^{2} r_{2} d^{2} r_{3} r_{12}^{2}}{r_{23}^{2} r_{31}^{2}}\left[\nabla_{1}^{2} \nabla_{2}^{2} \phi^{+}\left(y, r_{1}, r_{2}\right)\right] \cdot \phi\left(y, r_{2}, r_{3}\right) \phi\left(y, r_{3}, r_{1}\right)+(h . c) .
$$




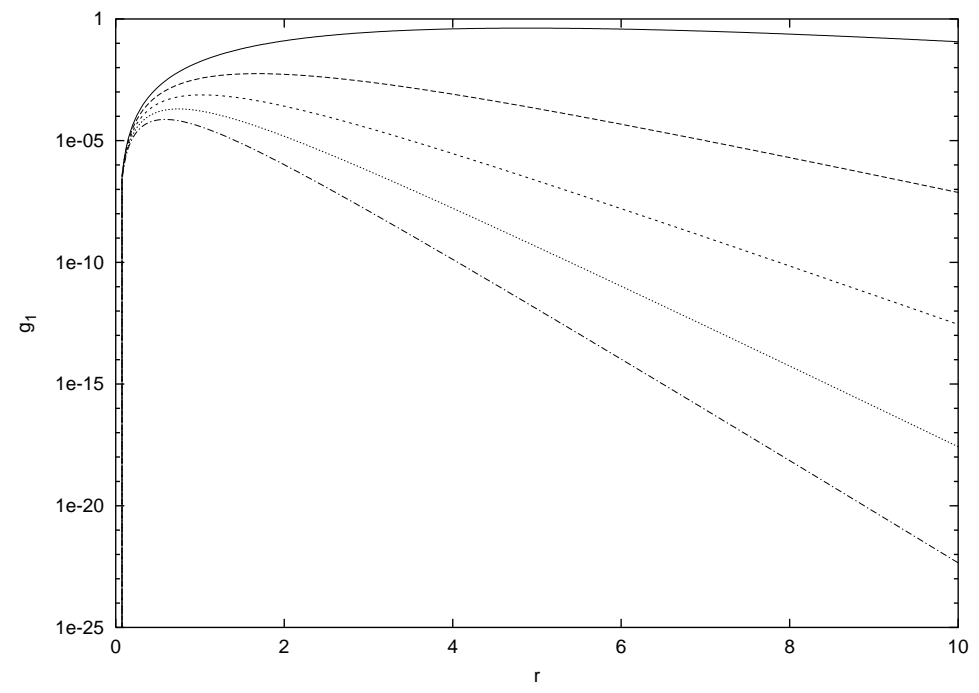

Figure 3: Pomeron propagator integrated over the final gluon coordinates $g_{1}(y, r)$ as a function of $r$. The initial BK function Eq. (64) is taken with $\gamma=0.1$. Curves from top to bottom correspond to $y=1,3,5,7$ and 9

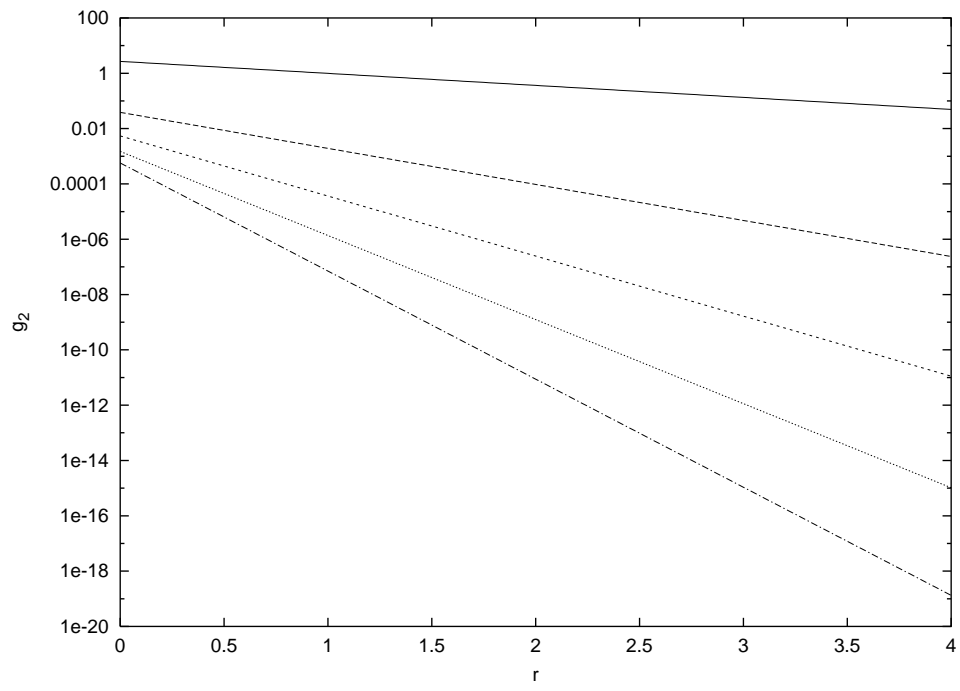

Figure 4: Pomeron propagator integrated over the initial gluon coordinates $g_{2}(y, r)$ as a function of $r$. The initial BK function Eq. (64) is taken with $\gamma=0.1$. Curves from top to bottom correspond to $y=1,3,5,7$ and 9 


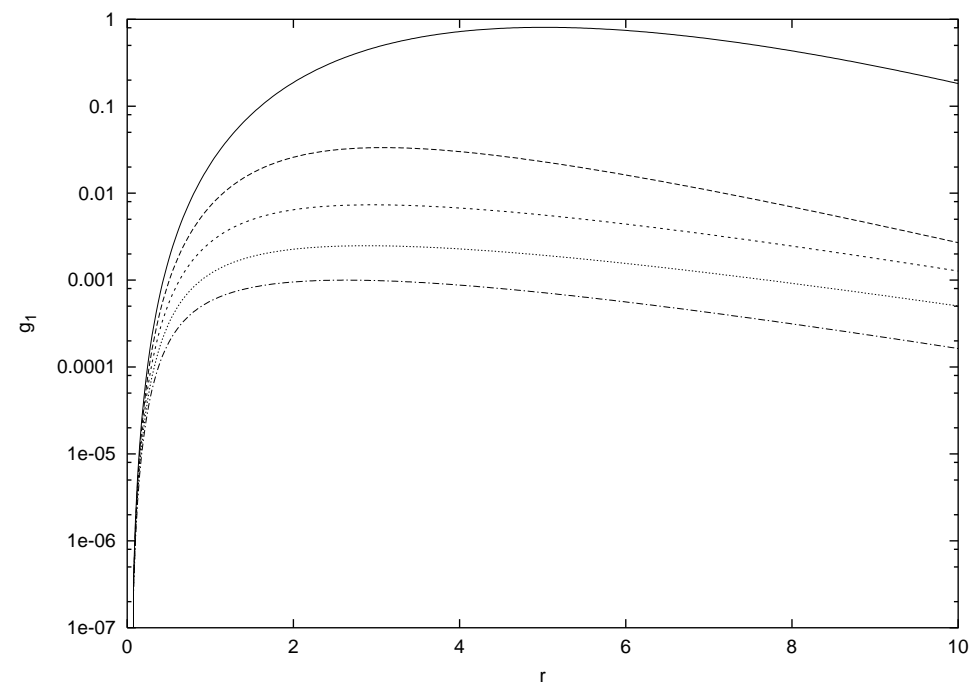

Figure 5: Same as Fig. 3 with $\gamma=2.0$ in the initial BK function Eq. (64) Curves from top to bottom correspond to $y=1,3,5,7$ and 9

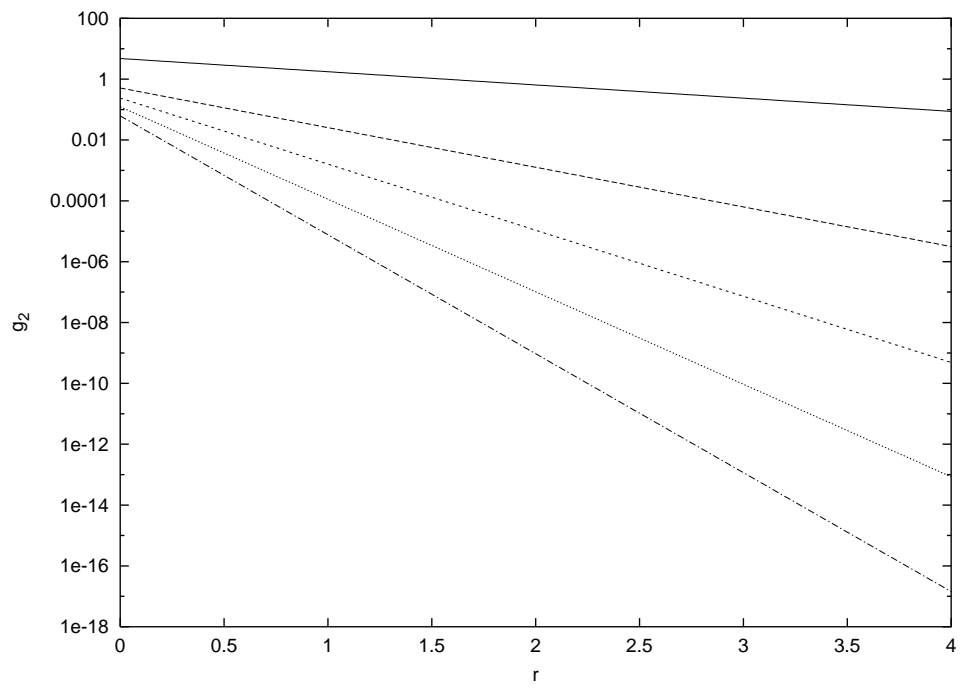

Figure 6: Same as Fig. 4 with $\gamma=2.0$ in the initial BK function Eq. (64) Curves from top to bottom correspond to $y=1,3,5,7$ and 9 


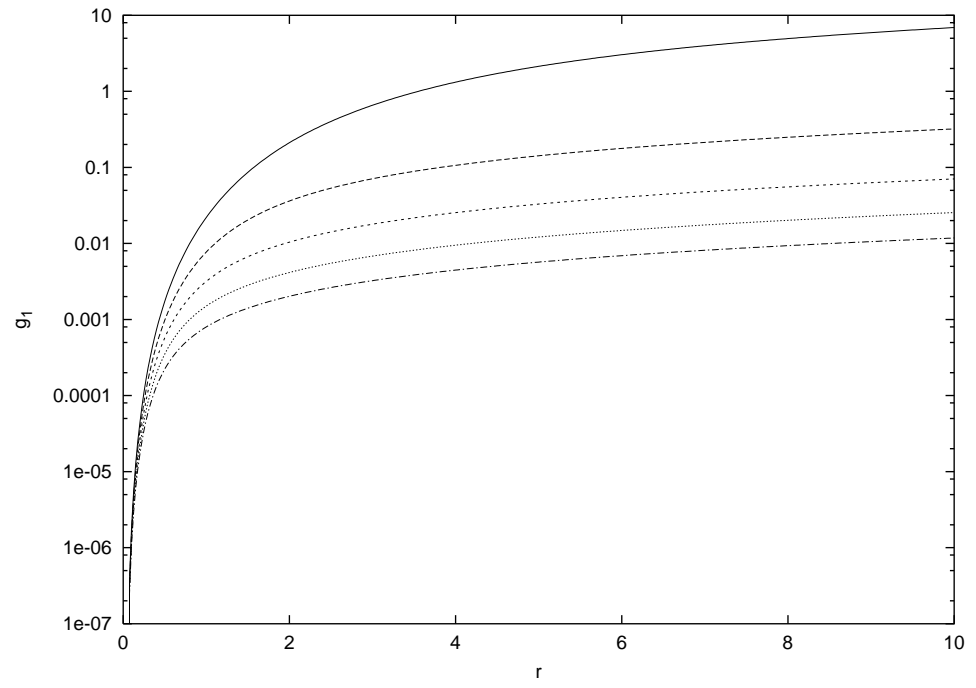

Figure 7: Same as Fig. 3 for the vacuum case. Curves from top to bottom correspond to $y=1$, $3,5,7$ and 9

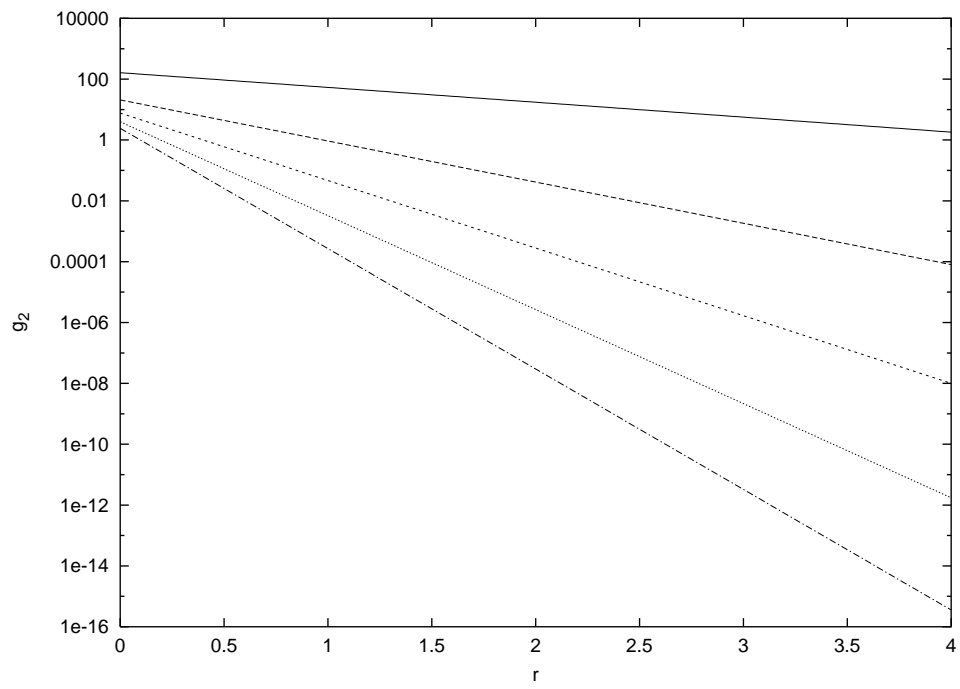

Figure 8: Same as Fig. 4 for the vacuum case. Curves from top to bottom correspond to $y=1$, $3,5,7$ and 9 


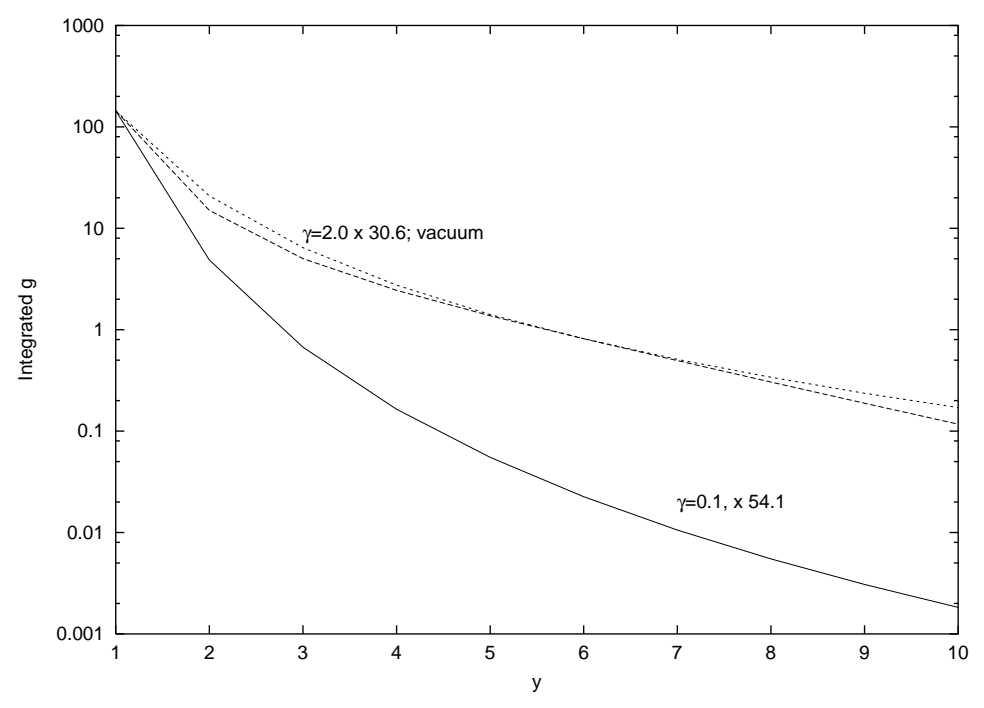

Figure 9: Fully integrated propagator $g(y, 0)$. The two curves with $\gamma=0.1$ (the lower one) and $\gamma=2.0$ (one of the two practically coinciding upper curves) are rescaled to have the same value with the vacuum curve (the other of the two upper curves) at $y=1$

Accordingly the pomeron self-mass $\Sigma$ is given by

$$
\Sigma\left(y, r_{2}, r_{1} \mid y^{\prime}, r_{2}^{\prime}, r_{1}^{\prime}\right)=-\left(\frac{2 \alpha_{s}^{2} N_{c}}{\pi}\right)^{2} \int \frac{d^{2} r_{3} r_{12}^{2}}{r_{23}^{2} r_{31}^{2}} \frac{d^{2} r_{3}^{\prime} r_{1^{\prime} 2^{\prime}}^{2}}{r_{2^{\prime} 3^{\prime}}^{2} r_{3^{\prime} 1^{\prime}}^{2}} g\left(y, r_{2}, r_{3} \mid y^{\prime}, r_{2}^{\prime}, r_{3}^{\prime}\right) g\left(y, r_{1}, r_{3} \mid y^{\prime}, r_{1}^{\prime}, r_{3}^{\prime}\right)
$$

(the minus sign reflects the absorptive character of the triple pomeron interaction).

Passing to $2+1$ dimensions we substitute the vertex function $r_{12}^{2} / r_{23}^{2} r_{13}^{2}$ by the difference of $\theta$ functions, as found in [6] and indicated in (2). The pomeron self-mass becomes

$$
\begin{gathered}
\Sigma\left(y, r_{2}, r_{1} \mid y^{\prime}, r_{2}^{\prime}, r_{1}^{\prime}\right)=-\left(8 \pi N_{c} \alpha_{s}^{2}\right)^{2} \int_{\min \left\{r_{2}, r_{1}\right\}}^{\max \left\{r_{2}, r_{1}\right\}} d r_{3} \int_{\min \left\{r_{2}^{\prime}, r_{1}^{\prime}\right\}}^{\max \left\{r_{2}^{\prime}, r_{1}^{\prime}\right\}} d r_{3}^{\prime} \\
g\left(y, r_{2}, r_{3} \mid y^{\prime}, r_{2}^{\prime}, r_{3}^{\prime}\right) g\left(y, r_{3}, r_{1} \mid y^{\prime}, r_{3}^{\prime}, r_{1}^{\prime}\right)
\end{gathered}
$$

It is trivial to see that this expression is zero due to the properties of the pomeron propagator. In fact take $r_{2}>r_{1}$ and $r_{2}^{\prime}>r_{1}^{\prime}$. The first propagator is different from zero only at $r_{3}^{\prime}>r_{3}$. and the second propagator is different from zero only at $r_{3}>r_{3}^{\prime}$. So their product is zero.

This circumstance is obvious since the interval where the propagator is different from zero is extending on both sides with the growth of $y$ as schematically shown in Fig. 10,a. Splitting the pomeron in two generates the picture shown in Fig. 10.b. It is evident that after splitting as the rapidity diminishes the two new pomerons can never interact with each other (points $r_{3}^{\prime}$ and $r_{3}^{\prime \prime}$ in Fig. 10,b cannot coincide). So loops do not exist in our theory.

\section{Conclusions}

In $2+1$ dimensions solutions of both the BK equation describing the nuclear field and BFKL equation in this field can be obtained in the closed form, expressed via infinite-dimensional matrices on the points dividing the coordinate interval. This makes it possible to find both the Green function of the BFKL equation and the pomeron propagator. It is important that the initial condition for the propagator at $y=y^{\prime}$ in principle admits addition of terms linear in one of the two final coordinates. We have proposed a choice which in our opinion is physically 


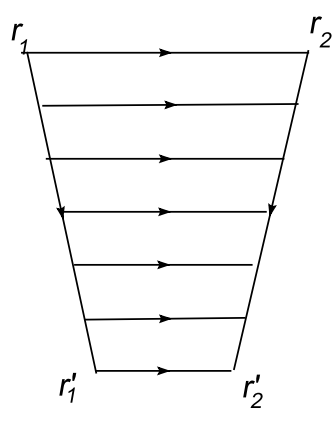

$A$

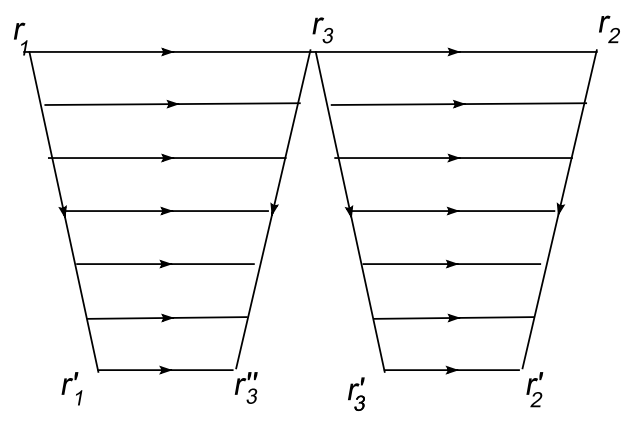

$B$

Figure 10: Schematic regions in the $(y, r)$ plane where the pomeron propagator (a) or a pair of propagators after splitting (b) are different from zero

motivated, since it has no infrared singularities at $y-y^{\prime}>0$. With this choice calculation of the propagator shows that the influence of the nuclear field very much depends on the behaviour of this field. If at large rapidities the nuclear $S$-matrix tends to zero (black disk limit) the field indeed makes the propagator fall with rapidity much faster than in the vacuum. On the other hand if the nuclear $S$-matrix tends to unity at high energies (zero interaction with the nucleus) the field in fact does not change the behaviour of the propagator in comparison to the nuclear case.

An important consequence of our choice of the propagator is that the region where it is different from zero does not change with energy and restricted to the interval of final coordinates which contains inside the one of the initial coordinates. As a result pomerons cannot form loops of any sort. Thus with this choice the quasi-classical solution of the BK equation gives the complete solution of the dipole interaction with the nucleus. The question if other choices of the propagator are admissible from some points of view remains open and will be considered in future studies.

\section{References}

[1] I.I Balitsky, Nucl. Phys. B 463 (1996) 99.

[2] Yu. V. Kovchegov, Phys. Rev. D 60 (1999) 034008; D 61 (2000) 074018.

[3] M.A.Braun, Phys. Lett. B 483 (2000) 115; B 632 (2006) 297.

[4] M.A.Braun, A.N.Tarasov, Eur. Phys. J. C 58 (2008) 383.

[5] M.A.Braun, A.N.Tarasov, Nucl. Phys. B 851 (2011) 533.

[6] J.Bartels, V.S.Fadin, L.N.Lipatov, Nucl. Phys. B 698 (2004) 255. 